\title{
Perfil epidemiológico do câncer infantil na Paraíba
}

\author{
Epidemiological profile of childhood cancer in thestate of Paraíba
}

\author{
Perfil epidemiológico del cáncer infantil en Paraíba
}

\begin{abstract}
Aline Tavares de Oliveira ${ }^{1}$, Milena Nunes Alves de Sousa ${ }^{1,2 *}$, Paula Christianne Gomes Gouveia Souto Maia ${ }^{1}$, André Luiz Dantas Bezerra ${ }^{2}$, Beatriz Coelho Pires de Moura ${ }^{2}$, Everson Vagner de Lucena Santos ${ }^{1,2}$, Gildenia Pinto dos Santos Trigueiro ${ }^{1}$, Tiago Bezerra de Sá de Sousa Nogueira ${ }^{1}$, Raquel Bezerra de Sá de Sousa Nogueira ${ }^{1}$, Miguel Aguila Toledo ${ }^{1}$, Vandezita Dantas de Medeiros Mazzaro $^{1}$, Larissa de Araújo Batista Suárez ${ }^{3}$, Patrícia Peixoto Custodio ${ }^{4,5}$, Ilana Andrade Santos do Egypto'.
\end{abstract}

\section{RESUMO}

Objetivo: Analisar o perfil epidemiológico do câncer infantil na Paraíba. Métodos: A investigação foi realizada mediante estudo documental, descritivo, transversal, com abordagem quantitativa. A coleta dos dados foi feita a partir das informações disponíveis na internet, no sitio do Instituto Nacional do Câncer. O instrumento utilizado para coleta de dados foi uma ficha contendo tipo de neoplasia, sexo e faixa etária mais acometida. Tais dados foram compilados no Microsoft Excel e sua análise foi efetivada mediante estatística descritiva. Resultados: Os achados indicaram que o tipo de câncer mais incidente nas crianças paraibanas, independente do sexo e com idade acima de um ano, corresponde às leucemias que são classificadas como grupo I; os do sistema nervoso central, grupo III e os linfomas, grupo II, respectivamente. Em relação ao grupo menor de um ano, observou-se que houve uma prevalência maior no sexo feminino nos grupos I, II, V, VI e IX, enquanto que no sexo masculino a maior prevalência foi encontrada no grupo III. Entretanto, é válido ressaltar que em relação ao grupo I, apesar de maior prevalência no sexo feminino, o grupo masculino também possui um valor considerável, com uma pequena diferença entre eles. Conclusão: A incidência dos mais variados tipos de tumores na população pediátrica é diferente em relação à faixa etária e ao sexo, tornando seu perfil epidemiológico bastante diversificado.

Palavras-Chave: Câncer, Pediatria, Diagnóstico Precoce.

\begin{abstract}
Objective: To analyze the epidemiological profile of childhood cancer in the state of Paraíba. Methods: The investigation was carried out by means of a documental, descriptive and transversal study, with a quantitative approach. The data collection was performed making using of the information available on the internet, on the National Cancer Institute website. The instrument used for the data collection was a form containing the type of neoplasm, gender and most affected age group. This data was compiled in Microsoft Excel and the analysis was carried out by means of descriptive statistics. Results: The findings indicated that the most incident type of cancer in children from the State of Paraíba, regardless of gender and over one year of age, corresponds to the leukemias, which are classified as group I; those of the central nervous system, group III and the lymphomas, group II, respectively. In relation to the group under one year of age, it was observed a higher prevalence in the female gender in the groups I, II, V, VI and IX, whereas in the male gender the highest prevalence was found in the group III. However, it is valid to point out that in relation to the group I, despite the higher prevalence in the female gender, the male group also had a considerable rate, with a small difference between them. Conclusion: The incidence of the most varied types of tumors in the pediatric population is different regarding age group and gender, making its epidemiological profile considerably diversified.
\end{abstract}

Keywords: Cancer, Pediatrics, Early Diagnosis.

${ }^{1}$ Curso de Medicina do Centro Universitário de Patos UNIFIP, Patos-PB. *E-mail: minualsa@hotmail.com

${ }^{2}$ Curso de Enfermagem da Faculdade Vale do Pajeú, São José do Egito-PE.

3 Departamento de Psicologia da Universidade Católica de Pernambuco, Recife-PE.

${ }^{4}$ Universidade Federal de Campina Grande, Cajazeiras-PB.

5 Universidade Federal de Campina Grande, Pombal-PB. 


\section{RESUMEN}

Objetivo: Analizar el perfil epidemiológico del cáncer infantil en Paraíba. Métodos: la investigación se llevó a cabo por el estudio documental, descriptivo, transversal, con enfoque cuantitativo. La recolección de datos fue hecha de la información disponible en internet, en lugar del Instituto Nacional del cáncer. El instrumento utilizado para la recolección de datos es un archivo que contiene el tipo de neoplasia, sexo y grupo de edad más afectado. Estos datos han sido recopilados en Microsoft Excel y el análisis se realizó mediante estadística descriptiva. Resultados: Los resultados indicaron que el tipo de incidente cáncer en los niños, independientemente del sexo y la edad más de un año, corresponde a que las leucemias se clasifican como grupo I; el sistema nervioso central, grupo III y linfomas, grupo II, respectivamente. En relación con el grupo de menos de un año, se observó que había una mayor prevalencia en las mujeres en los grupos I, II, V, VI y IX, mientras que en varones la prevalencia más alta se encontró en el grupo III. Sin embargo, es válido destacar que en comparación con el grupo I, aunque más frecuente en la mujer, el grupo masculino también tiene un valor considerable, con una pequeña diferencia entre ellos. Conclusión: la incidencia de varios tipos de tumores en la población pediátrica es diferente en relación a edad y sexo, haciendo su muy diverso perfil epidemiológico.

Palabras clave: Cáncer, Pediatría, Diagnóstico Precoz.

\section{INTRODUÇÃO}

O câncer infantil está relacionado a um grupo de várias doenças que possuem em comum a proliferação exacerbada e descontrolada de células anormais cujo local de ocorrência no organismo pode ser o mais variado. Os tumores, que podem ser definidos como o conjunto dessas células anormais, mais frequentes entre as faixas etárias de zero a 19 anos são as leucemias (que afetam os glóbulos brancos do sangue), os linfomas (sistema linfático) e os do sistema nervoso central (RIBEIRO LL, SILVA LE e FRANÇA AMB, 2016).

A ocorrência desse tipo de doença consiste em um evento que sensibiliza profundamente a família e a vida familiar em diversas esferas. Mudanças na rotina, assim como dos papéis e dos relacionamentos entre os membros da família, incerteza generalizada, problemas no trabalho, crises conjugais, dificuldades financeiras e uma série de privações e restrições no cotidiano são alguns exemplos de desafios que são impostos a todos os membros (MENDES-CASTILLO ANC e BOUSSO RS., 2016).

Conforme o Instituto Nacional do Câncer (INCA, 2017), é importante a orientação aos pais que ao sinal de alguma anormalidade na criança é preciso levá-la a um médico, se possível, um pediatra para uma avaliação, já que na maioria das vezes, os sintomas referidos estão relacionados a patologias comuns nessa faixa etária. Além disso, a criança ou o adolescente pode estar em boas condições de saúde, aparentemente, no início da doença. São sinais e sintomas comuns, pele descorada, presença de hematomas e/ou sangramentios, dor nos ossos, inchaços ou caroços, perda de peso, febre, falta de ar e tosse persistente, alterações oculares, com pupila esbranquçada, vômitos, dores de cabeça, letargia, fadiga, tontura e outros.

Atualmente, para o diagnóstico e seguimento da evolução do câncer infantil, são usados vários exames de imagem dos mais complexos até os mais simples, como: ressonância magnética, tomografia computadorizada, ultrassonografia e radiografia convencional. Além de exames laboratoriais e bioquímicos como os marcadores tumorais auxiliando no diagnóstico e evolução da doença (RIBEIRO LL, SILVA LE e FRANÇA AMB, 2016).

Por sua vez, o câncer infantil (de 0 a 19 anos) é considerado raro quando comparado com o câncer em outras faixas etárias, correspondendo a $2 \%$ e $3 \%$ de todos os cânceres registrados no Brasil. Há uma tendência, em crianças e adolescentes, a presença de menores períodos de latência, pois este costuma proliferar-se rapidamente e tornar-se bastante invasivo, porém possui melhor resposta à quimioterapia (INCA, 2017).

Portanto, conhecer o perfil epidemiológico do câncer infantil é fundamental. Fundamentando-se na assertiva, o objetivo do trabalho inclui, além de analisar o perfil epidemiológico do câncer infantil na Paraíba, analisar os principais tipos de câncer infantil relacionados com a idade e o sexo dos pacientes e ratificar a importância da instalação de um centro de tratamento de câncer infantil no Sertão Paraibano. 


\section{MÉTODOS}

Pesquisa do tipo documental, descritiva, transversal, com abordagem quantitativa conduzida a partir de bancos de dados disponíveis na Internet. O estudo contemplou os dados epidemiológicos do estado da Paraíba e as informações foram obtidas no site do Instituto Nacional do Câncer (INCA).

A Paraíba possui uma população de 3.766 .528 habitantes de acordo com o último censo do Instituto Brasileiro de Geografia e Estatística (IBGE, 2017) feito no ano de 2010 e uma população estimada em 4.025.558 habitantes para o ano de 2017.

A população estudada se restringiu ao universo infantil, compreendendo a faixa etária de zero a 19 anos, cadastradas nos bancos de dados disponíveis na Internet. A incidência dos mais variados tipos de tumores na população pediátrica é diferente em relação à faixa etária e ao sexo mais acometido. Os tumores são classificados em 12 grupos. O I compreende as leucemias; o II, os linfomas; o III, os do sistema nervoso central; o IV do sistema nervoso simpático; o V, os retinoblastomas; o VI, os tumores renais. O VII, os tumores hepáticos; o VIII, os tumores ósseos; o IX, os sarcomas de partes moles; o X, as neoplasias de células germinativas, trofoblásticas e outras gonadais; o XI, os carcinomas e outras neoplasias malignas epiteliais; e o XII, os outros tumores malignos e não especificados. Foram considerados os dados existentes mais atuais, cujos pacientes se adequaram a faixa etária determinada, sem restrição de sexo. E foram excluídos os dados incompletos.

A coleta dos dados foi executada no mês de abril de 2018. Os dados foram compilados no Microsoft Excel. A análise dos dados, por sua vez, foi efetivada mediante estatística descritiva.

\section{RESULTADOS}

Os dados disponíveis no INCA mais recentes são referentes ao ano de 2008 e os valores são referentes às taxas de incidência, por 1 milhão de crianças e adolescentes. Conforme o Gráfico 1, em relação ao grupo menor de um ano, cujo perfil difere das faixas etárias maiores, observaram-se prevalências maiores no sexo feminino nos grupos I, II, V, VI e IX, enquanto que no sexo masculino a maior prevalência foi encontrada no grupo III. Entretanto, é válido ressaltar que em relação ao grupo I, apesar de maior prevalência no sexo feminino, o grupo masculino também possui um valor considerável, com uma pequena diferença entre eles.

Gráfico 1 - Incidência dos grupos de tumores em crianças menores de um ano.

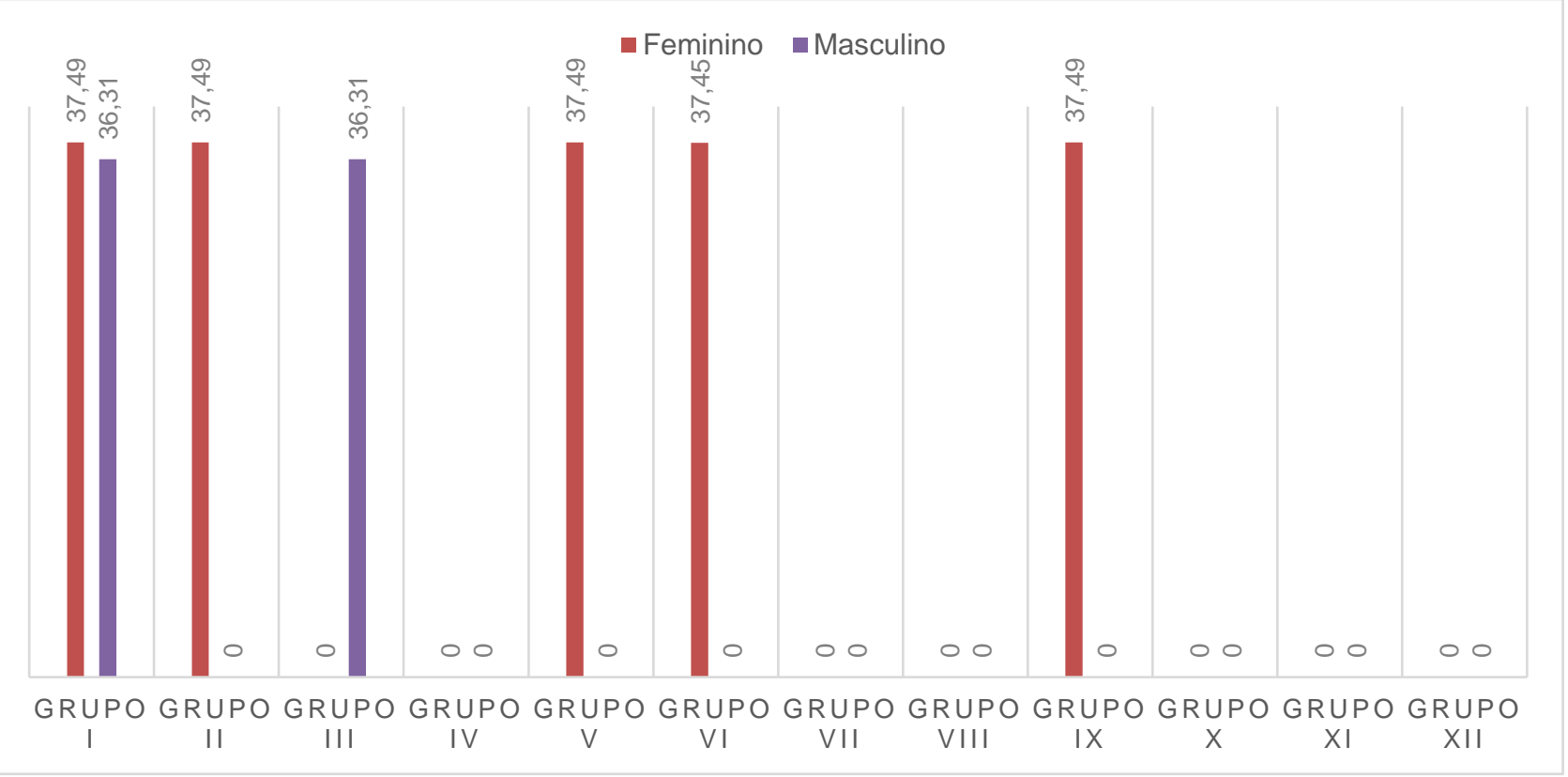

Nota: Os valores do gráfico são absolutos.

Fonte: INCA (2008). 
Considerando a Tabela 1, é possível verificar a incidência dos grupos de tumores em relação às seguintes faixas etárias: 1-4 anos, 5-9 anos, 10-14 anos e 15-19 anos, para ambos os sexos. A partir dos achados, o sexo masculino foi mais acometido nos grupos I, II, IV, V e VIII; o feminino nos grupos VI, XIX e XI.

Tabela 1 - Incidência dos grupos de tumores em relação à idade e ao sexo acometido.

\begin{tabular}{|c|c|c|c|c|}
\hline Grupos & 1-4 anos & 5-9 anos & $10-14$ anos & 15-19 anos \\
\hline $\begin{array}{l}\text { Grupo I } \\
\text { Feminino } \\
\text { Masculino }\end{array}$ & $\begin{array}{l}55,43 \\
44,75\end{array}$ & $\begin{array}{l}34,66 \\
68,33\end{array}$ & $\begin{array}{l}12,71 \\
12,71\end{array}$ & $\begin{array}{l}22,65 \\
35,29\end{array}$ \\
\hline $\begin{array}{l}\text { Grupo II } \\
\text { Feminino } \\
\text { Masculino }\end{array}$ & $\begin{array}{l}9,24 \\
17,9\end{array}$ & $\begin{array}{l}6,93 \\
13,67\end{array}$ & $\begin{array}{l}31,77 \\
25,43\end{array}$ & $\begin{array}{l}16,99 \\
35,29\end{array}$ \\
\hline $\begin{array}{l}\text { Grupo III } \\
\text { Feminino } \\
\text { Masculino }\end{array}$ & $\begin{array}{l}18,48 \\
35,8\end{array}$ & $\begin{array}{l}13,86 \\
13,67 \\
\end{array}$ & $\begin{array}{l}38,13 \\
6,36 \\
\end{array}$ & $\begin{array}{l}4,5 \\
5,88 \\
\end{array}$ \\
\hline $\begin{array}{l}\text { Grupo IV } \\
\text { Feminino } \\
\text { Masculino }\end{array}$ & $\begin{array}{l}9,24 \\
26,85 \\
\end{array}$ & $\begin{array}{l}0 \\
0 \\
\end{array}$ & $\begin{array}{l}0 \\
0 \\
\end{array}$ & $\begin{array}{l}4,58 \\
0 \\
\end{array}$ \\
\hline $\begin{array}{l}\text { Grupo V } \\
\text { Feminino } \\
\text { Masculino }\end{array}$ & $\begin{array}{l}9,24 \\
17,9 \\
\end{array}$ & $\begin{array}{l}0 \\
0\end{array}$ & $\begin{array}{l}0 \\
0\end{array}$ & $\begin{array}{l}0 \\
0\end{array}$ \\
\hline $\begin{array}{l}\text { Grupo VI } \\
\text { Feminino } \\
\text { Masculino }\end{array}$ & $\begin{array}{l}36,95 \\
8,95 \\
\end{array}$ & $\begin{array}{l}0 \\
6,85 \\
\end{array}$ & $\begin{array}{l}0 \\
0\end{array}$ & $\begin{array}{l}4,5 \\
0 \\
\end{array}$ \\
\hline $\begin{array}{l}\text { Grupo VII } \\
\text { Feminino } \\
\text { Masculino }\end{array}$ & $\begin{array}{l}0 \\
0\end{array}$ & $\begin{array}{l}0 \\
0\end{array}$ & $\begin{array}{l}0 \\
0\end{array}$ & $\begin{array}{l}0 \\
0\end{array}$ \\
\hline $\begin{array}{l}\text { Grupo VIII } \\
\text { Feminino } \\
\text { Masculino }\end{array}$ & $\begin{array}{l}0 \\
0 \\
\end{array}$ & $\begin{array}{l}6,93 \\
20,5 \\
\end{array}$ & $\begin{array}{l}12,71 \\
19,07 \\
\end{array}$ & $\begin{array}{l}22,65 \\
64,07 \\
\end{array}$ \\
\hline $\begin{array}{l}\text { Grupo IX } \\
\text { Feminino } \\
\text { Masculino }\end{array}$ & $\begin{array}{l}18,48 \\
17,9 \\
\end{array}$ & $\begin{array}{l}13,86 \\
0\end{array}$ & $\begin{array}{l}19,06 \\
0\end{array}$ & $\begin{array}{l}11,33 \\
17,65 \\
\end{array}$ \\
\hline $\begin{array}{l}\text { Grupo X } \\
\text { Feminino } \\
\text { Masculino }\end{array}$ & $\begin{array}{l}0 \\
8,95\end{array}$ & $\begin{array}{l}6,93 \\
0 \\
\end{array}$ & $\begin{array}{l}12,71 \\
0\end{array}$ & $\begin{array}{l}0 \\
5,88 \\
\end{array}$ \\
\hline $\begin{array}{l}\text { Grupo XI } \\
\text { Feminino } \\
\text { Masculino }\end{array}$ & $\begin{array}{l}0 \\
0\end{array}$ & $\begin{array}{l}6,93 \\
0\end{array}$ & $\begin{array}{l}6,35 \\
0\end{array}$ & $\begin{array}{l}62,3 \\
17,65\end{array}$ \\
\hline $\begin{array}{l}\text { Grupo XII } \\
\text { Feminino } \\
\text { Masculino }\end{array}$ & $\begin{array}{l}0 \\
17,9\end{array}$ & $\begin{array}{l}6,93 \\
0\end{array}$ & $\begin{array}{l}6,35 \\
6,36\end{array}$ & $\begin{array}{l}16,99 \\
0\end{array}$ \\
\hline
\end{tabular}

Nota: Os valores do gráfico são absolutos.

Fonte: INCA (2008).

\section{DISCUSSÃO}

A pesquisa demonstrou que o tipo de câncer mais incidente nas crianças paraibanas, independente do sexo e com idade acima de um ano, corresponde às leucemias que são classificadas como grupo I; os do sistema nervoso central, grupo III e os linfomas, grupo II, respectivamente. Tais dados corroboram com os existentes na literatura (SANCHES FLFZ, et al., 2015; HANNA LMO, et al., 2016; NEGREIROS AALV, et al., 2015).

Os tipos de câncer infantis mais comuns entre infantes são as leucemias, principalmente a leucemia linfoide aguda (LLA) e a leucemia mieloide aguda (LMA), tumor no sistema nervoso central, linfoma, neuroblastoma, tumor de Wilms, osteossarcoma e sarcoma de Ewing, nessa ordem de acometimento (SANCHES FLFZ, et al., 2015). 
Ao refletir-se sobre as leucemias, reconhece-se que o tipo agudo é considerado uma neoplasia heterogênea que afeta a medula óssea, em que os constituintes normais medulares e do sangue são substituídos por blastos, células consideradas imaturas, ainda estes blastos podem se acumular em outros tecidos (SANCHES FLFZ, et al., 2015). Essas doenças malignas da infância afetam as células do sistema hematopoiético e os tecidos de sustentação e, por serem predominantemente de natureza embrionária, são feitas de células indiferenciadas (HANNA LMO, et al., 2016). É importante ressaltar que em menores de um ano, a incidência é praticamente a mesma em ambos os sexos.

Por conseguinte, os tumores do sistema nervoso central (SNC) representam o segundo grupo de diagnóstico mais comum - 17\% a $25 \%$ das neoplasias na infância -, além de ser o tumor sólido mais frequente e a principal causa de mortalidade por câncer em jovens (NEGREIROS AALV, et al., 2015). Correspondem a um grande grupo de neoplasias diferenciando-os quanto a localização primária, o tipo histológico e o comportamento (LIMA ER, et al., 2015). Em relação aos menores de um ano, foi observada incidência apenas no sexo masculino.

O tratamento varia de acordo com suas características e também conforme fatores inerentes ao paciente, tais como a idade; e baseia-se no uso isolado ou associado de cirurgia, radioterapia e quimioterapia. Apesar de todos os avanços obtidos nas últimas décadas, ainda representam as neoplasias com maior índice de mortalidade em Oncologia Pediátrica (LIMA ER, et al., 2015).

Os linfomas, classificados como grupo II, são neoplasias do tecido linfoide e são divididos em linfoma de Hodgkin (LH) e linfoma não Hodgkin (LNH). Encontra-se como o terceiro tipo de câncer mais incidente em crianças, após as leucemias e os tumores cerebrais. Sua incidência se eleva com a idade, representando $3 \%$ dos cânceres em crianças com idade inferior a cinco anos e $24 \%$ dos casos de câncer em adolescentes com idade entre 15 e 19 anos (OLIVEIRA MCLA e CAMPOS MK, 2015). Em menores de um ano, a pesquisa demonstrou incidência apenas no sexo feminino.

O estudo constatou que o câncer do grupo $V$ que corresponde aos retinoblastomas, acomete mais o sexo masculino, com proporção de dois meninos para cada menina, na primeira infância, ou seja, de um a quatro anos, não havendo relato nas outras faixas etárias. O retinoblastoma é o tumor intraocular primário mais comum e têm recebido cada vez mais atenção, principalmente, nos países em desenvolvimento $(\mathrm{Hu} \mathrm{H}$, et al., 2018). Em menores de um ano, a incidência é apenas no sexo feminino.

Quanto ao grupo VI, dos tumores renais, a pesquisa revelou que embora nas idades de um a quatro e de 15 a 19 anos haja maior incidência no sexo feminino e que entre cinco e nove anos a predominância seja no sexo masculino, quando se trata de menores de um ano a incidência é apenas no sexo feminino. Em relação ao grupo VIII, quanto aos tumores ósseos, foi constatada incidência maior no sexo masculino a partir dos cinco anos, predominando-se até os 19 anos. A investigação radiológica dos tumores ósseos é muitas vezes um achado incidental nos pacientes assintomáticos, devendo ser conduzido com cuidado e consideração especializada. Nos casos em que há sintomas, como a dor, a radiografia simples pode ser utilizada como um recurso complementar de diagnóstico essencial para análise do quadro (ANDRADE NETO F, et al., 2016).

O grupo IX, os sarcomas de partes moles, acomete mais o sexo feminino, a partir de cinco anos com incidência decrescente a partir dos 15. O osteossarcoma, um dos representantes da categoria, é um tipo de câncer com característica intensa de agressividade, e suas células têm o potencial gerar matriz óssea, podendo ser identificada em várias etapas da vida, entretanto a incidência desta é maior em crianças e adolescentes devido ao período de crescimento ósseo acelerado na puberdade (ALBARADO KVP, MEDEIROS HP e TEIXEIRA E, 2015). Em menores de um ano, a incidência é apenas no sexo feminino.

Os carcinomas e outras neoplasias malignas epiteliais, grupo XI, acometem mais o sexo feminino, a partir de 5 anos. Enquanto que no sexo masculino, a incidência ocorre apenas no intervalo de 15 a 19 anos, mas em proporções menores. Um dos integrantes desse agrupamento é o câncer de pele e esse período da vida é considerado crítico já que compreende em torno de $80 \%$ da exposição solar durante a vida. Além disso, a exposição solar prematura apresenta maior relevância no desenvolvimento das neoplasias cutâneas do que a exposição mais tardia, e a fotoproteção a partir dos primeiros anos de vida é eficaz de forma a reduzir de 
forma significativa o risco de melanoma (STERN RS, WEINSTEIN MC e BAKER SG, 1986; GODAR E, et al., 2003). Por fim, oportuno citar as limitações deste estudo, que se centraram na falta de atualidade dos dados, pois os mais atuais datavam do ano de 2008 e não contemplavam todos os municípios do estado da Paraíba, apenas os da capital João Pessoa, o que pode evidenciar o fato de haver poucos estudos e pesquisas em relação ao tema nas demais regiões do estado, sendo, portanto, de extrema relevância.

\section{CONCLUSÃO}

A incidência dos mais variados tipos de tumores na população pediátrica é diferente em relação à faixa etária e ao sexo, tornando seu perfil epidemiológico bastante diversificado. Contudo, os achados indicaram que as leucemias (grupo I) são o tipo de câncer mais incidente nas crianças paraibanas em idade superior a um ano e independente do sexo. Também, se destacaram as neoplasias do sistema nervoso central (grupo III) e os linfomas (grupo II), respectivamente. Quanto ao grupo menor de um ano, os grupos I, II, V, VI e IX foram prevalentes no sexo feminino e as neoplasias do grupo III no masculino.

\section{REFERÊNCIAS}

1. ALBARADO KVP, MEDEIROS HP, TEIXEIRA E. Acting child nursing care with osteosarcoma and Ewing's sarcoma: experience report. Revista de Enfermagem da Ufpi, 2015;4(4):100-104.

2. ANDRADE NETO $F$, et al. Ensaios iconográficos tumores ósseos do joelho: achados na radiologia convencional. Radiologia Brasileira, 2016; 49(3):182-189.

3. GODAR E, et al. UV Doses of Young Adults. Photochemistry and Photobiology, 2003; 77: 453-457.

4. $\mathrm{HU} \mathrm{H}$, et al. Characterization, treatment and prognosis of retinoblastoma with central nervous system metastasis. $B M C$ Ophthalmology, 2018; 18(1);107-107.

5. HANNA LMO, et al. Pediatric cancer: epidemiologic profile of attended children in a reference center in thestate of Para. Journal Of Research In Destistry, 2016; 4(6); 100-107.

6. INSTITUTO BRASILEIRO DE GEOGRAFIA E ESTATíSTICA (IBGE). Panorama da Paraíba. Disponível em: <https://cidades.ibge.gov.br/brasil/pb/panorama>. Acesso em: 8 nov. 2017.

7. INSTITUTO NACIONAL DE CÂNCER (INCA). Câncer Infantil. Disponível em: <http://www2.inca.gov.br/wps/wcm/connect/tiposdecancer/site/home/infantil>. Acesso em: 18 out. 2017.

8. LIMA ER, et al. Análise de sobrevida de pacientes portadores de tumores do sistema nervoso central. Revista Médica de Minas Gerais, 2015; 25(6):10-16.

9. MENDES-CASTILLO ANC, BOUSSO RS. A experiência das avós de crianças com câncer. Revista Brasileira de Enfermagem, 2016; 69(3): 559-565.

10. NEGREIROS AALV, et al. Aspectos Epidemiológicos dos Tumores do Sistema Nervoso Central Pediátricos em um Hospital de Referência de João Pessoa (PB) entre 2009 e 2011. Revista Acadêmica do Centro de Ciência Médicas da Universidade Federal da Paraíba, 2015;1(1): 51-59

11. OLIVEIRA MCLA, CAMPOS MK. Linfomas em Pediatria. Pediatria Moderna, 2015; 51(5): 173-180.

12. RIBEIRO LL, SILVA LE, FRANÇA AMB. Cuidados paliativos à criança portadora de doença oncológica. Ciências Biológicas e da Saúde, 2016; 3(30: 151-164.

13. SANCHES FLFZ, et al. Comparison of biochemical and immunological profile of pediatric patients with acute myeloid leukemia in relation to healthy individuals. Jornal de Pediatria (Rio J), 2015;91(5):478--484.

14. STERN RS, WEINSTEIN MC, BAKER SG. Risk reduction for nonmelanoma skin cancer with childhood sunscreen use. Archives of Dermatology, 1986; 122: 537-545. 